\title{
Well-posedness and stability results for the Gardner equation
}

\author{
Miguel A. Alejo
}

\begin{abstract}
In this article we present local well-posedness results in the classical Sobolev space $H^{s}(\mathbb{R})$ with $s>1 / 4$ for the Cauchy problem of the Gardner equation, overcoming the problem of the loss of the scaling property of this equation. We also cover the energy space $H^{1}(\mathbb{R})$ where global well-posedness follows from the conservation laws of the system. Moreover, we construct solitons of the Gardner equation explicitly and prove that, under certain conditions, this family is orbitally stable in the energy space.
\end{abstract}

Mathematics Subject Classification (2010). Primary 35Q51, 35Q53;

Secondary 49K40, 47J35.

Keywords. Gardner equation, Local well posedness, Integrability, Stability.

\section{Introduction}

In this article we present some results about the regularity for the Cauchy problem of the Gardner Equation (shortly GE)

$$
\left\{\begin{array}{l}
v_{t}+v_{x x x}+6 \sigma\left(v^{2}\right)_{x}+2\left(v^{3}\right)_{x}=0 \quad \sigma, t, x \in \mathbb{R} \\
v(x, 0)=v_{0}(x) \in H^{s}(\mathbb{R})
\end{array}\right.
$$

with data in the classical Sobolev space $H^{s}(\mathbb{R})$ and the orbital stability of its soliton solutions. In a previous work [1] we faced the problem of finding some $L^{\infty}$-solutions with nonzero limits at infinity and with geometrical interpretation of the focusing modified Korteweg-de Vries (shortly mKdV)

$$
u_{t}+u_{x x x}+2\left(u^{3}\right)_{x}=0,
$$

that is, solutions of the type $\sigma+v(x+c t)$, with $c>0, \sigma \in \mathbb{R}$ and $v$ a traveling wave solution with exponential decay at infinity. When introducing such ansatz in the focusing $\mathrm{mKdV}$ (1.2), the GE (1.1) appears (up to rescaling). This evolution equation is characterized to be composed by a $\mathrm{KdV}$ term $\left(v^{2}\right)_{x}$ 
and a positive mKdV term $\left(v^{3}\right)_{x}$. The competition between these two different nonlinear terms together with the linear dispersive term $v_{x x x}$ allows the existence of more intricate soliton solutions (see Sect. 3) as well as exact breather solutions (see [1]). The Gardner equation plays also an important role in the proof of the $L^{2}$-stability of the multisoliton solution of $\mathrm{KdV}$, through to the so called Gardner transform which links $H^{1}$-solutions of the Gardner equation with $L^{2}$-solutions of the $\mathrm{KdV}$ equation (see [2]).

Soliton solutions (or equivalently $\sigma$-soliton solutions of the $\mathrm{mKdV}$, if their asymptotic constant is equal to $\sigma$ ) in the focusing and defocusing cases $( \pm$ sign respectively in the cubic nonlinearity of (1.2)) are easily related through the transformation of the asymptotic parameter $\sigma$ to $i \sigma$. Indeed, these solitons can be explicitly obtained integrating the resulting second order ODE which arises when we look for traveling wave solutions of (1.1) of the type $v(x+c t)$ with $c>0$ (see Sect. 3).

In this paper we are also interested to prove the orbital stability á la Zhidkov of these soliton solutions under small perturbations in $H^{1}(\mathbb{R})$. Hence, we need a global well posedness (GWP for short) result for the initial value problem (IVP for short) (1.1) in the energy space $H^{1}(\mathbb{R})$, and therefore it is enough for our aim to prove the local well posedness (LWP for short) of the IVP (1.1) below the conservation law $H^{1}(\mathbb{R})$. Indeed, we prove the LWP in $H^{s}(\mathbb{R})$ with $s>1 / 4$. Note that the Gardner equation is not scaling invariant. This loss of the scale property raises two problems. The first one appears in the proof of persistence of the solution in the LWP result since the scale property can not be used and we need introduce a rescaling of the problem in terms of a new auxiliar function. The second problem is that the proof of the convexity condition suggested by P. Zhidkov can not be deduced directly and we need to integrate the Lyapunov functional. As a consequence, we see that the proof of the stability of solitons in the focusing and defocusing cases are almost identical. Therefore, we will only show here the focusing case.

The LWP of the IVP for KdV with initial data in $H^{s}(\mathbb{R}), s>-3 / 4$ was obtained by Kenig, Ponce and Vega in [11]. They showed sharp bilinear estimates in the functional space $X^{s, b}$, introduced by Bourgain in [5], up to the index $s=-3 / 4$. In [6], Christ, Colliander and Tao proved the LWP of the IVP for $\mathrm{KdV}$ with initial data in $H^{s}(\mathbb{R}), s \geq-3 / 4$, using a modified Miura transform and the existence theory for the $\mathrm{mKdV}$. They also proved the global theory for initial data in $H^{s}(\mathbb{R}), s>-3 / 4$.

The LWP of the IVP for the focusing $\mathrm{mKdV}$ with initial data in the Sobolev space $H^{s}(\mathbb{R}), s \geq 1 / 4$, was given by Kenig, Ponce and Vega in [9], where they also proved the global well posedness in the energy space $H^{s}(\mathbb{R}), s \geq 1$. The global result below the conservation law was shown, for initial data in $H^{s}(\mathbb{R}), s>1 / 4$ by Colliander, Keel, Staffilani, Takaoka and Tao in [7], using the existence theory for KdV and the Miura transform.

In the next sections we present the local well-posedness of the IVP for the GE (1.1) in the Sobolev space $H^{s}(\mathbb{R}), s>1 / 4$, obtaining bilinear and trilinear estimates by using the auxiliar space of functions $X^{s, b}(\mathbb{R} \times \mathbb{R})$ and the $[k ; \mathbb{R}]$-multiplier theory introduced by Tao [13]. We also cover the energy 
space $H^{1}(\mathbb{R})$ where global well posedness follows from the conservation laws of the problem. Moreover, we construct solitons of the GE and we prove, under certain conditions, that this family is orbitally stable in $H^{1}(\mathbb{R})$.

\section{Local theory}

We are interested in the local (in time, $t=T>0$ ) existence theory for the IVP of the GE (1.1) with initial data $\operatorname{in}^{1} H^{1 / 4^{+}}(\mathbb{R})$. Since the GE is not invariant under scaling transformations, we can not proceed as usual, i.e., prove the LWP of (1.1) at time $t=1$ and use the scaling transformation to extent the local time to $t=T>0$. So we devise a procedure closely related to the usual one, introducing an auxiliar function $w$ through a scaling transformation. Next, we prove the LWP at $t=1$ of the associated GE for $w$ at a certain scaling parameter $\lambda$ (to be determined). Finally we use this result and the scaling relation to obtain directly the LWP of the IVP for the GE (1.1) at $t=T>0$.

We make the following scaling transformation:

$$
v(x, t)=\lambda^{\alpha} w(\lambda x, \lambda t), \quad \lambda>0, \alpha>2,
$$

where $v$ is a solution of the IVP for the GE (1.1), so that the associated Gardner equation for the auxiliar function $w$ is

$$
\left\{\begin{array}{l}
w_{t}+w_{x x x}+6 \sigma \lambda^{\alpha-2}\left(w^{2}\right)_{x}+2 \lambda^{2(\alpha-1)} \\
\left(w^{3}\right)_{x}=0, \quad \sigma, \lambda>0, \alpha, t, x \in \mathbb{R} \\
w(x, 0)=w_{0}(x) \in H^{s}(\mathbb{R})
\end{array}\right.
$$

For $s, b \in \mathbb{R}$, the space of functions $X^{s, b}$ denotes the completion of the Schwartz space $\mathcal{S}\left(\mathbb{R}^{2}\right)$ with respect to the norm

$$
\|f\|_{X^{s, b}}=\left(\int_{-\infty}^{+\infty} \int_{-\infty}^{+\infty}\left(1+\left|\tau-\xi^{3}\right|\right)^{2 b}(1+|\xi|)^{2 s}|\hat{f}(\xi, \tau)|^{2} d \xi d \tau\right)^{1 / 2} .
$$

We invoke the work of Tao [13] about multilinear estimates to define a $[k ; \mathbb{R}]$ multiplier as any function $m: \Gamma_{k}(\mathbb{R}) \rightarrow \mathbb{C}$, where $\Gamma_{k}(\mathbb{R})$ is the hyperplane

$$
\Gamma_{k}(\mathbb{R}):=\left\{\left(\xi_{1}, \xi_{2}, \ldots, \xi_{k}\right) \in \mathbb{R}^{k}: \xi_{1}+\xi_{2}+\cdots+\xi_{k}=0\right\},
$$

endowed with the measure

$$
\int_{\Gamma_{k}(\mathbb{R})} f:=\int_{\mathbb{R}^{k-1}} f\left(\xi_{1}, \ldots, \xi_{k-1},-\xi_{1}-\cdots-\xi_{k}-1\right) d \xi_{1} d \xi_{2} \ldots d \xi_{k-1} .
$$

For $\|m\|_{[k ; \mathbb{R}]}$ we denote the best constant such that the inequality

$$
\left|\int_{\Gamma_{k}(\mathbb{R})} m(\xi) \prod_{j=1}^{k} f_{j}\left(\xi_{j}\right)\right| \leq\|m\|_{[k ; \mathbb{R}]} \prod_{j=1}^{k}\left\|f_{j}\right\|_{L^{2}(\mathbb{R})},
$$

\footnotetext{
${ }^{1}$ In what follows, $H^{1 / 4^{+}}(\mathbb{R})$ means $H^{s}(\mathbb{R})$ with $s>1 / 4$.
} 
holds for all test functions $f_{i}$ on $\mathbb{R}$. In the sequel, the free operator $W(t)$ denotes an element of the unitary group $\{W(t)\}_{-\infty}^{+\infty}$ describing the solution of the linear IVP associated to (1.1)

$$
\left\{\begin{array}{l}
v_{t}+v_{x x x}=0, \quad t, x \in \mathbb{R} \\
v(x, 0)=v_{0}(x),
\end{array}\right.
$$

where

$$
v(x, t)=W(t) v_{0}(x)=\int_{\mathbb{R}} e^{i x \xi+t \xi^{3}} \hat{v}_{0}(\xi) d \xi .
$$

Finally, let $\psi \in C_{0}^{\infty}(\mathbb{R})$ with $\psi \equiv 1$ on $[-1 / 2,1 / 2]$ and $\operatorname{supp} \psi \subseteq(-1,1)$. Then, our main results are:

Theorem 2.1. Let be $s>1 / 4$ and $\sigma>0$. Then there exist constants $d_{1}>$ $0, d_{2}>0, \lambda>0$ and $b \in(1 / 2,1)$, such that for all $w_{0} \in H^{s}(\mathbb{R})$, exists a unique solution $w \in C\left([-1,1]: H^{s}(\mathbb{R})\right)$ of $(2.2)$ with $\lambda$ and $w$ satisfying

$$
\begin{aligned}
& \lambda \leq \min \left(d_{1}\left\|w_{0}\right\|_{H^{s}}^{\frac{-1}{\alpha-2}}, d_{2}\left\|w_{0}\right\|_{H^{s}}^{\frac{-1}{\alpha-1}}\right), \\
& w \in C\left([-1,1]: H^{s}(\mathbb{R})\right), \\
& w \in X^{s, b} \subseteq L_{x, l o c}^{p}\left(\mathbb{R}: L_{t}^{2}([-1,1])\right), \quad 1 \leq p \leq \infty, \\
& \partial_{x}\left(w^{3}\right) \in X^{s, b-1}(\mathbb{R} \times \mathbb{R}) \wedge \quad \partial_{x}\left(w^{2}\right) \in X^{s, b-1}(\mathbb{R} \times \mathbb{R}) .
\end{aligned}
$$

Theorem 2.2. Let be $s>1 / 4$ and $\sigma>0$. Then there exists $b \in(1 / 2,1)$, such that for every $v_{0}(x) \in H^{s}(\mathbb{R})$, there exist a local time $T=T\left(\left\|v_{0}\right\|_{H^{s}}\right)>0$ (with $T(\rho) \rightarrow \infty$ when $\rho \rightarrow 0$ ) and a unique solution $v(x, t) \equiv v(t)$ of (1.1) such that $\sigma+v(t)$ is the unique solution of (1.2) in the interval $[-T, T]$ satisfying

$$
\begin{aligned}
& v \in C\left([-T, T]: H^{s}(\mathbb{R})\right), \\
& v \in X^{s, b} \subseteq L_{x, l o c}^{p}\left(\mathbb{R}: L_{t}^{2}([-T, T])\right), \quad 1 \leq p \leq \infty, \\
& \partial_{x}\left(v^{3}\right) \in X^{s, b-1}(\mathbb{R} \times \mathbb{R}) \wedge \quad \partial_{x}\left(v^{2}\right) \in X^{s, b-1}(\mathbb{R} \times \mathbb{R}) .
\end{aligned}
$$

We will resort to the following preliminary estimates for the free operator $W(t)$, of the unitary group describing the solution of the linear IVP associated to (1.1) (for proofs of such estimates, see [10]):

Lemma 2.3. Let $s>1 / 4,1 / 2<b<1$ and $0<\delta<1$. Then, there exists $a$ constant $c>0$ such that:

1. $\left\|\psi\left(\delta^{-1} t\right) W(t) v_{0}\right\|_{X^{s, b}} \leq c \delta^{\frac{1-2 b}{2}}\left\|v_{0}\right\|_{H^{s}}$.

2. $\left\|\psi\left(\delta^{-1} t\right) h\right\|_{X^{s, b}} \leq c \delta^{\frac{1-2 b}{2}}\|h\|_{X^{s, b}}$.

3. $\left\|\psi\left(\delta^{-1} t\right) \int_{0}^{t} W\left(t-t^{\prime}\right) w\left(t^{\prime}\right) d t^{\prime}\right\|_{X^{s, b}} \leq c \delta^{\frac{1-2 b}{2}}\|w\|_{X^{s, b-1}}$.

4. $\left\|\psi\left(\delta^{-1} t\right) \int_{0}^{t} W\left(t-t^{\prime}\right) w\left(t^{\prime}\right) d t^{\prime}\right\|_{H^{s}} \leq c \delta^{\frac{1-2 b}{2}}\|w\|_{X^{s, b-1}}$.

Moreover, to prove the local theorem we will need the following bilinear and trilinear estimates:

Lemma 2.4. Let $s>1 / 4$. Then, for all $u_{i}=\psi(t) \phi_{i}(x, t), i=1,2$, with support in $\mathbb{R} \times[-1,1]$ and $b=1 / 2+\epsilon, 0<\epsilon \ll 1$, the following inequality holds:

$$
\left\|u_{1} u_{2}\right\|_{L^{2}(\mathbb{R} \times \mathbb{R})} \leq c|| \phi_{1}\left\|_{X^{s, b}(\mathbb{R} \times \mathbb{R})}\right\| \phi_{2} \|_{X^{-1 / 2,1-b}(\mathbb{R} \times \mathbb{R})} .
$$


Proof. For (2.16) see Proposition 6.2. in [13].

Lemma 2.5. Let $s>1 / 4$ and $b=1 / 2+\epsilon, 0<\epsilon \ll 1$. Then, for all $u_{i}=$ $\psi(t) \phi_{i}(x, t), i=1,2,3$, with support in $\mathbb{R} \times[-1,1]$, and $c>0$, the following inequality holds

$$
\left\|\partial_{x}\left(u_{1} u_{2} u_{3}\right)\right\|_{X^{s, b-1}(\mathbb{R} \times \mathbb{R})} \leq c\left\|\phi_{1}\right\|_{X^{s, b}(\mathbb{R} \times \mathbb{R})}\left\|\phi_{2}\right\|_{X^{s, b}(\mathbb{R} \times \mathbb{R})}\left\|\phi_{3}\right\|_{X^{s, b}(\mathbb{R} \times \mathbb{R})} .
$$

Proof. ${ }^{2}$ We emphasize that essentially the proof appears in the work of Tao [13] (it is worth to note that it also works for $s=1 / 4$ ), but we present here for the sake of completeness. We summarize the proof in three steps:

1. By the duality of the spaces $X^{s, b-1}(\mathbb{R} \times \mathbb{R}), X^{-s, 1-b}(\mathbb{R} \times \mathbb{R})$ and using Plancherel, we obtain that

$$
\begin{aligned}
& \left.\int_{\mathbb{R}} \int_{\mathbb{R}} \bar{f} \partial_{x}\left(u_{1} u_{2} u_{3}\right) d x d t=\int_{\mathbb{R}} \int_{\mathbb{R}} \hat{\bar{f}}(-\xi,-\tau) \partial_{x} \widehat{\left(u_{1} u_{2}\right.} u_{3}\right)(\xi, \tau) d \xi d \tau \\
& \quad=\int_{\mathbb{R}} \int_{\mathbb{R}}(i \xi) \hat{\bar{f}}(-\xi,-\tau) \widehat{u_{1} u_{2} u_{3}}(\xi, \tau) d \xi d \tau
\end{aligned}
$$

Then, taking into account that $\xi_{3}=\xi-\xi_{1}-\xi_{2}, \tau_{3}=\tau-\tau_{1}-\tau_{2}$, the expression (2.18) reduces to

$$
\begin{aligned}
& \int_{\mathbb{R}} \int_{\mathbb{R}} \bar{f} \partial_{x}\left(u_{1} u_{2} u_{3}\right) d x d t \\
& \quad=\int_{\Gamma_{3+1}(\mathbb{R} \times \mathbb{R})} i\left(\xi_{1}+\xi_{2}+\xi_{3}\right) \cdot\left(\prod_{j=1}^{3} \hat{u}_{j}\left(\xi_{j}, \tau_{j}\right)\right) \cdot \hat{\bar{f}}\left(\xi_{4}, \tau_{4}\right) .
\end{aligned}
$$

Resorting to Lemma 2.3 (2) and in terms of the $X^{s, b}$ norm, we have

$$
\begin{aligned}
& \left|\int_{\Gamma_{3+1}(\mathbb{R} \times \mathbb{R})}\left(\xi_{1}+\xi_{2}+\xi_{3}\right) \cdot\left(\prod_{j=1}^{3} \hat{u}_{j}\left(\xi_{j}, \tau_{j}\right)\right) \cdot \hat{\bar{f}}\left(\xi_{4}, \tau_{4}\right)\right| \\
& \leq c \mid \frac{\left(\xi_{1}+\xi_{2}+\xi_{3}\right)\left\langle\xi_{4}\right\rangle^{s}\left\langle\tau_{4}-\xi_{4}^{3}\right\rangle^{b-1}}{\prod_{j=1}^{3}\left\langle\xi_{j}\right\rangle^{s}\left\langle\tau_{j}-\xi_{j}^{3}\right\rangle^{b}} \|_{[3+1 ; \mathbb{R} \times \mathbb{R}]} \\
& \quad \cdot\left(\prod_{j=1}^{3}\left\|\phi_{j}\right\|_{X^{s, b}}\right)\|f\|_{X^{-s, 1-b}} .
\end{aligned}
$$

So, denoting $b=1 / 2+\epsilon$, for $0<\epsilon \ll 1$, it is enough to prove that

$$
\left\|\frac{\left(\xi_{1}+\xi_{2}+\xi_{3}\right)\left\langle\xi_{4}\right\rangle^{s}\left\langle\tau_{4}-\xi_{4}^{3}\right\rangle^{b-1}}{\prod_{j=1}^{3}\left\langle\xi_{j}\right\rangle^{s}\left\langle\tau_{j}-\xi_{j}^{3}\right\rangle^{b}}\right\|_{[3+1 ; \mathbb{R} \times \mathbb{R}]} \lesssim 1 .
$$

\footnotetext{
${ }^{2}$ In this proof we follow the notation of [13]. Here $\hat{\imath}$ denotes the Fourier transform.
} 
2. Since $\xi_{4}=-\xi_{1}-\xi_{2}-\xi_{3}$ implies $\left|\xi_{1}+\xi_{2}+\xi_{3}\right| \sim \xi_{4}$, and applying the inequality

$$
\left\langle\xi_{4}\right\rangle^{s+1} \lesssim\left\langle\xi_{4}\right\rangle^{1 / 2} \sum_{j=1}^{3}\left\langle\xi_{j}\right\rangle^{s+1 / 2}
$$

(2.21) simplifies as follows:

$$
\begin{aligned}
& \left\|\frac{\left(\xi_{1}+\xi_{2}+\xi_{3}\right)\left\langle\xi_{4}\right\rangle^{s}\left\langle\tau_{4}-\xi_{4}^{3}\right\rangle^{b-1}}{\prod_{j=1}^{3}\left\langle\xi_{j}\right\rangle^{s}\left\langle\tau_{j}-\xi_{j}^{3}\right\rangle^{b}}\right\|_{[3+1 ; \mathbb{R} \times \mathbb{R}]} \sim\left\|\frac{\left\langle\xi_{4}\right\rangle^{s+1}\left\langle\tau_{4}-\xi_{4}^{3}\right\rangle^{b-1}}{\prod_{j=1}^{3}\left\langle\xi_{j}\right\rangle^{s}\left\langle\tau_{j}-\xi_{j}^{3}\right\rangle^{b}}\right\|_{[3+1 ; \mathbb{R} \times \mathbb{R}]} \\
& \lesssim\left\|\frac{\left\langle\xi_{4}\right\rangle^{1 / 2} \sum_{i=1}^{3}\left\langle\xi_{i}\right\rangle^{s+1 / 2}\left\langle\tau_{4}-\xi_{4}^{3}\right\rangle^{b-1}}{\prod_{j=1}^{3}\left\langle\xi_{j}\right\rangle^{s}\left\langle\tau_{j}-\xi_{j}^{3}\right\rangle^{b}}\right\|_{[3+1 ; \mathbb{R} \times \mathbb{R}]} .
\end{aligned}
$$

3. Assuming now (w.l.g.) that dual variable $\xi_{2}$ is the greater one, we use the following estimate:

$$
\frac{\sum_{j=1}^{3}\left\langle\xi_{j}\right\rangle^{s+1 / 2}}{\left\langle\xi_{2}\right\rangle^{s}} \lesssim\left\langle\xi_{2}\right\rangle^{1 / 2}
$$

Then (2.23) remains as

$$
\left\|\frac{\left\langle\xi_{4}\right\rangle^{1 / 2}\left\langle\xi_{2}\right\rangle^{1 / 2}\left\langle\xi_{1}\right\rangle^{-s}\left\langle\xi_{3}\right\rangle^{-s}\left\langle\tau_{4}-\xi_{4}^{3}\right\rangle^{b-1}}{\left\langle\tau_{2}-\xi_{2}^{3}\right\rangle^{1-b} \prod_{i=1}^{2}\left\langle\tau_{2 i-1}-\xi_{2 i-1}^{3}\right\rangle^{b}}\right\|_{[3+1 ; \mathbb{R} \times \mathbb{R}]} .
$$

Selecting $m$ as

$$
m\left(\xi_{1}, \xi_{2}\right)=\frac{\left\langle\xi_{1}\right\rangle^{-s}\left\langle\xi_{2}\right\rangle^{1 / 2}}{\left\langle\tau_{2}-\xi_{2}^{3}\right\rangle^{1-b}\left\langle\tau_{1}-\xi_{1}^{3}\right\rangle^{b}},
$$

it is possible to rewrite $(2.24)$ as

$$
\left\|m\left(\xi_{1}, \xi_{2}\right) \cdot \overline{m\left(-\xi_{3},-\xi_{4}\right)}\right\|_{[3+1 ; \mathbb{R} \times \mathbb{R}]}=\left\|m\left(\xi_{1}, \xi_{2}\right)\right\|_{[2+1 ; \mathbb{R} \times \mathbb{R}]}^{2}
$$

where we have used the estimate $T T^{*}$ given in [13, p.8]. In concluding, we need that

$$
\left\|m\left(\xi_{1}, \xi_{2}\right)\right\|_{[2+1 ; \mathbb{R} \times \mathbb{R}]}=\left\|\frac{\left\langle\xi_{1}\right\rangle^{-s}\left\langle\xi_{2}\right\rangle^{1 / 2}}{\left\langle\tau_{2}-\xi_{2}^{3}\right\rangle^{1-b}\left\langle\tau_{1}-\xi_{1}^{3}\right\rangle^{b}}\right\|_{[2+1 ; \mathbb{R} \times \mathbb{R}]} \lesssim 1,
$$

which is proved in Lemma 2.4.

By using the same steps than in the proof of (2.17), it is straightforward to prove the following:

Lemma 2.6. Let $s>0$ and $b=1 / 2+\epsilon, 0<\epsilon \ll 1$. Then for all $u_{i}=$ $\psi(t) \phi_{i}(x, t), i=1,2$, with support in $\mathbb{R} \times[-1,1]$, and $c>0$, the following inequality holds:

$$
\left\|\partial_{x}\left(u_{1} u_{2}\right)\right\|_{X^{s, b-1}(\mathbb{R} \times \mathbb{R})} \leq c\left\|\phi_{1}\right\|_{X^{s, b}(\mathbb{R} \times \mathbb{R})}\left\|\phi_{2}\right\|_{X^{s, b}(\mathbb{R} \times \mathbb{R})} .
$$


Proof of Theorem 2.1. We denote the $H^{s}(\mathbb{R})$ norm of the initial data $w_{0}(x)$ as

$$
\left\|w_{0}\right\|_{H^{s}}=r_{w}
$$

For $w_{0} \in H^{s}(\mathbb{R})$ with $s>1 / 4$, the localized Duhamel operator is

$$
\begin{aligned}
\Phi_{1, w_{0}}(w) \equiv & \Phi_{1}(w) \\
= & \psi(t) W(t) w_{0}-6 \sigma \lambda^{\alpha-2} \psi(t) \int_{0}^{t} W\left(t-t^{\prime}\right) \partial_{x}\left[\left(\psi\left(t^{\prime}\right) w\left(t^{\prime}\right)\right)^{2}\right] d t^{\prime} \\
& -2 \lambda^{2(\alpha-1)} \psi(t) \int_{0}^{t} W\left(t-t^{\prime}\right) \partial_{x}\left[\left(\psi\left(t^{\prime}\right) w\left(t^{\prime}\right)\right)^{3}\right] d t^{\prime} .
\end{aligned}
$$

Then, the proof of the theorem is summarized in four steps. Indeed, in the first two we prove that $\Phi_{1}$ is a contraction in the following ball of $X^{s, b}(\mathbb{R} \times \mathbb{R})$ :

$$
\mathcal{B} \equiv \mathcal{B}\left(3 c_{0} r_{w}\right):=\left\{w \in X^{s, b}:\|w\|_{X^{s, b}} \leq 3 c_{0} r_{w}\right\}
$$

1. If $w \in \mathcal{B}, \alpha>2$ and combining (1), (3), (2.6), (2.5), the following inequalities hold:

$$
\begin{aligned}
\left\|\Phi_{1}(w)\right\|_{X^{s, b}} \leq & c_{0}\left\|w_{0}\right\|_{H^{s}}+c_{1} \sigma \lambda^{\alpha-2}\left\|\psi(t)^{2} \partial_{x}\left(w^{2}(x, t)\right)\right\|_{X^{s, b-1}} \\
& +c_{2} \lambda^{2(\alpha-1)}\left\|\psi(t)^{3} \partial_{x}\left(w^{3}(x, t)\right)\right\|_{X^{s, b-1}} \\
\leq & c_{0}\left\|w_{0}\right\|_{H^{s}}+c \cdot c_{1} \sigma \lambda^{\alpha-2}\|w(x, t)\|_{X^{s, b}}^{2} \\
& +c \cdot c_{2} \lambda^{2(\alpha-1)}\|w(x, t)\|_{X^{s, b}}^{3} \\
\leq & c_{0} r_{w}+c \cdot c_{1} \sigma \lambda^{\alpha-2}\left(3 c_{0} r_{w}\right)^{2}+c \cdot c_{2} \lambda^{2(\alpha-1)}\left(3 c_{0} r_{w}\right)^{3} \\
\leq & c_{0} r_{w}\left\{1+c_{0} \lambda^{\alpha-2} r_{w}+c_{0}^{2} \lambda^{2(\alpha-1)} r_{w}^{2}\right\} \\
\leq & 3 c_{0} r_{w}
\end{aligned}
$$

where the last inequality is verified by choosing $\lambda$ which satisfy the following two conditions:

$$
\begin{aligned}
r_{w} \lambda^{\alpha-2} c_{0} & \leq 1 / 4, \\
r_{w}^{2} \lambda^{2(\alpha-1)} c_{0}^{2} & \leq 1 / 4 .
\end{aligned}
$$

Then, if we select $\lambda_{0}$ as the minimum value of (2.30) and choose $\lambda$ as

$$
\begin{aligned}
& \lambda \leq \lambda_{0}=\min \left(d_{1}\left\|w_{0}\right\|_{H^{s}}^{\frac{-1}{\alpha-2}}, \quad d_{2}\left\|w_{0}\right\|_{H^{s}}^{\frac{-1}{(\alpha-1)}}\right), \\
& d_{1}=\left(\frac{1}{4 c_{0}}\right)^{\frac{1}{\alpha-2}}, \quad d_{2}=\left(\frac{1}{4 c_{0}^{2}}\right)^{\frac{1}{2(\alpha-1)}}, \quad \alpha>2 .
\end{aligned}
$$

the conditions (2.30) will be satisfied. In concluding,

$$
\Phi_{1}(\mathcal{B}) \subseteq \mathcal{B}
$$


2. With the same ideas as above (that is, combining (1), (2), (3), (2.6), (2.5 and $(2.29))$, if $w, \tilde{w} \in \mathcal{B}$ then we have

$$
\begin{aligned}
\left\|\Phi_{1}(w)-\Phi_{1}(\tilde{w})\right\|_{X^{s, b}} \leq c_{0} \sigma \lambda^{\alpha-2}\left\|\psi(t)^{2} \partial_{x}\left(w^{2}(x, t)-\tilde{w}^{2}(x, t)\right)\right\|_{X^{s, b-1}} \\
\quad+c \lambda^{2(\alpha-1)}\left\|\psi(t)^{3} \partial_{x}\left(w^{3}(x, t)-\tilde{w}^{3}(x, t)\right)\right\| \|_{X^{s, b-1}} \\
=c_{0} \sigma \lambda^{\alpha-2}\left\|\psi(t)^{2} \partial_{x}[(w(x, t)-\tilde{w}(x, t))(w(x, t)+\tilde{w}(x, t))]\right\|_{X^{s, b-1}} \\
\quad+c \lambda^{2(\alpha-1)} \| \psi(t)^{3} \partial_{x} \\
\quad\left[(w(x, t)-\tilde{w}(x, t))\left(w(x, t)^{2}+\tilde{w}^{2}(x, t)+w(x, t) \tilde{w}(x, t)\right)\right] \|_{X^{s, b-1}}
\end{aligned}
$$

Now taking into account

(i) Bilinear estimate: given $u_{1}=w-\tilde{w}, u_{2}=w+\tilde{w}$, we have, using the bilinear estimate (see Lemma 2.6)

$$
\begin{aligned}
& \left\|\psi(t)^{2} \partial_{x}\left(w^{2}(x, t)-\tilde{w}^{2}(x, t)\right)\right\|_{X^{s, b-1}}=\left.\left\|\psi(t)^{2} \partial_{x}\left[u_{1} u_{2}\right]\right\|\right|_{X^{s, b-1}} \\
& \quad \leq\left. c_{1}\left\|u_{1}\right\|\right|_{X^{s, b}}\left\|u_{2}\right\| X_{X^{s, b}} \leq c_{1}\|w-\tilde{w}\|_{X^{s, b}}\left\{\|w\|_{X^{s, b}}+\|\tilde{w}\|_{X^{s, b}}\right\} \\
& \quad \leq 3 \cdot 2 c_{0} c_{1} \cdot r_{w}\|w-\tilde{w}\|_{X^{s, b}} .
\end{aligned}
$$

(ii) Trilinear estimate: given $u_{1}=w-\tilde{w}, v_{1}=w, v_{2}=\tilde{w}, u_{2}=w+\tilde{w}$, we have

$$
\begin{aligned}
& \left\|\psi(t)^{3} \partial_{x}\left(w^{3}(x, t)-\tilde{w}^{3}(x, t)\right)\right\|_{X^{s, b-1}} \\
& \quad=\left\|\psi(t)^{3} \partial_{x}\left[(w-\tilde{w})\left(w^{2}+\tilde{w}^{2}+w \tilde{w}\right)\right]\right\| \|_{X^{s, b-1}} \\
& \quad=\left\|\psi(t)^{3} \partial_{x}\left[(w-\tilde{w})(w+\tilde{w})^{2}-(w-\tilde{w}) w \tilde{w}\right]\right\|_{X^{s, b-1}} \\
& \quad=\left\|\psi(t)^{3} \partial_{x}\left[u_{1} u_{2} u_{2}\right]-\psi(t)^{3} \partial_{x}\left[u_{1} v_{1} v_{2}\right]\right\|_{X^{s, b-1}} .
\end{aligned}
$$

and using the trilinear estimate (see Lemma 2.5), we obtain

$$
\begin{aligned}
& \left\|\psi(t)^{3} \partial_{x}\left(w^{3}(x, t)-\tilde{w}^{3}(x, t)\right)\right\|_{X^{s, b-1}} \\
& \quad=\left\|\psi(t)^{3} \partial_{x}\left[u_{1} u_{2} u_{2}\right]-\psi(t)^{3} \partial_{x}\left[u_{1} v_{1} v_{2}\right]\right\|_{X^{s, b-1}} \\
& \quad \leq c_{2}\left\|u_{1}\right\|_{X^{s, b}}\left\|u_{2}\right\|_{X^{s, b}}^{2}+\left.c_{2}\left\|u_{1}\right\|_{X^{s, b}}\left\|v_{1}\right\|\right|_{X^{s, b}}\left\|v_{2}\right\| X_{X^{s, b}} \\
& \quad \leq c_{2}\left\|u_{1}\right\|_{X^{s, b}}\left\{\left(\|w\|_{X^{s, b}}+\|\tilde{w}\|_{X^{s, b}}\right)^{2}+\|w\|_{X^{s, b}}\|\tilde{w}\|_{X^{s, b}}\right\} \\
& \quad \leq c_{2}\|w-\tilde{w}\|_{X^{s, b}}\left\{2\left(3 c_{0} \cdot r_{w}\right)^{2}+\left(3 c_{0} \cdot r_{w}\right)^{2}\right\} \\
& \quad=27 c_{0}^{2} c_{2} \cdot r_{w}^{2}\|w-\tilde{w}\|_{X^{s, b}} .
\end{aligned}
$$

Hence (2.34) simplifies as

$$
\begin{aligned}
& \left\|\Phi_{1}(w)-\Phi_{1}(\tilde{w})\right\|_{X^{s, b}} \leq 6 c c_{0} c_{1} \cdot \sigma \lambda^{\alpha-2} r_{w}\|w-\tilde{w}\|_{X^{s, b}} \\
& \quad+27 c c_{0}^{2} c_{2} \lambda^{2(\alpha-1)} r_{w}^{2}\|w-\tilde{w}\|_{X^{s, b}} \\
& \leq\left(c_{0} \lambda^{\alpha-2} r_{w}+c_{0}^{2} \lambda^{2(\alpha-1)} r_{w}^{2}\right)\|w-\tilde{w}\|_{X^{s, b}} \\
& \leq \frac{1}{2}\|w-\tilde{w}\|_{X^{s, b}}
\end{aligned}
$$

where the last inequality holds whenever we choose $\lambda$ less than the minimum value $\lambda_{0}$ in (2.31). Therefore, $\Phi_{1}$ is a contraction in $\mathcal{B}$ and then, there exists a unique $w \in \mathcal{B}\left(3 c_{0} r_{w}\right)$ which satisfies 


$$
\begin{gathered}
\psi(t) w(t)=\psi(t)\left\{W(t) w_{0}-6 \sigma \lambda^{\alpha-2} \int_{0}^{t} W\left(t-t^{\prime}\right) \partial_{x}\left[\left(\psi\left(t^{\prime}\right) w\left(t^{\prime}\right)\right)^{2}\right] d t^{\prime}\right. \\
\left.-2 \lambda^{2(\alpha-1)} \int_{0}^{t} W\left(t-t^{\prime}\right) \partial_{x}\left[\left(\psi\left(t^{\prime}\right) w\left(t^{\prime}\right)\right)^{3}\right] d t^{\prime}\right\}
\end{gathered}
$$

and in the temporal interval $[-1,1], w(\cdot)$ is solution of the IVP $(2.2)$. Recall that with the same argument as in (2.37) it is also possible to prove that

$$
\|w-\tilde{w}\|_{X^{s, b}} \leq c\left\|w_{0}-\tilde{w}_{0}\right\|_{H^{s}} .
$$

3. Before proving the property of persistence (2.13), we must show that the solution $w$ goes to the initial data $w_{0}$ in the classical $H^{s}$ norm, when $t \rightarrow 0$. In this way, we must check that the integral terms on the right of the following inequality go to 0 when $t \rightarrow 0$ :

$$
\begin{gathered}
\left\|w(t)-\psi(t) W(t) w_{0}\right\|_{H^{s}} \leq 6 \sigma \lambda^{\alpha-2}\left\|\psi(t) \int_{0}^{t} W\left(t-t^{\prime}\right) \partial_{x}\left[\left(\psi\left(t^{\prime}\right) w\left(t^{\prime}\right)\right)^{2}\right] d t^{\prime}\right\|_{H^{s}} \\
\quad+2 \lambda^{2(\alpha-1)}\left\|\psi(t) \int_{0}^{t} W\left(t-t^{\prime}\right) \partial_{x}\left[\left(\psi\left(t^{\prime}\right) w\left(t^{\prime}\right)\right)^{3}\right] d t^{\prime}\right\|_{H^{s}},
\end{gathered}
$$

To this end, we rewrite such terms in order to apply Lemma 2.3 (4) and analyze the behavior when $\eta \rightarrow 0$ of the following general expression:

$$
\left\|\psi(\eta) \int_{0}^{\eta} W\left(\eta-t^{\prime}\right) F\left(\cdot, t^{\prime}\right) d t^{\prime}\right\|_{H^{s}} .
$$

Taking $t^{\prime \prime}=\frac{t^{\prime}}{\eta}$, recalling $t^{\prime \prime} \equiv t^{\prime}$ and with the change (w.l.g.) of the cut off function from $\psi(\eta)$ to $\psi(1)$, the expression (2.41) is rewritten as follows:

$$
\begin{aligned}
& \left\|\psi(1) \int_{0}^{1} W\left(\eta\left(1-t^{\prime}\right)\right) F\left(\cdot, \eta t^{\prime}\right) \eta d t^{\prime}\right\|_{H^{s}} \\
& =\left(\int\left|\psi(1) \int_{0}^{1} W\left(\eta\left(1-t^{\prime}\right)\right)\left(D^{s} F\left(\cdot, \eta t^{\prime}\right)\right)(x) \eta d t^{\prime}\right|^{2} d x\right)^{1 / 2} .
\end{aligned}
$$

With the change of variable $x=\eta^{1 / 3} y$, we rewrite (2.42) as

$$
\begin{aligned}
& \left(\int\left|\psi(1) \int_{0}^{1} W\left(\eta\left(1-t^{\prime}\right)\right)\left(D^{s} F\left(\cdot, \eta t^{\prime}\right)\right)\left(\eta^{1 / 3} y\right) \eta d t^{\prime}\right|^{2} \eta^{1 / 3} d y\right)^{1 / 2} \\
& =\eta^{1+\frac{1}{6}}\left(\int\left|\psi(1) \int_{0}^{1} W\left(\eta\left(1-t^{\prime}\right)\right)\left(D^{s} F\left(\cdot, \eta t^{\prime}\right)\right)\left(\eta^{1 / 3} y\right) d t^{\prime}\right|^{2} d y\right)^{1 / 2}
\end{aligned}
$$

Now, we analyze the integrand $W\left(\eta\left(1-t^{\prime}\right)\right)\left(D^{s} F\left(\cdot, \eta t^{\prime}\right)\right)\left(\eta^{1 / 3} y\right)$ of $(2.43)$. For that, we define

$$
G_{1}\left(y, \eta t^{\prime}\right)=D^{s} F\left(\eta^{1 / 3} y, \eta t^{\prime}\right) \equiv D^{s} F_{\eta}\left(y, t^{\prime}\right), \quad \text { where } F_{\eta}\left(y, t^{\prime}\right)=F\left(\eta^{1 / 3} y, \eta t^{\prime}\right) .
$$

In this way, $W\left(1-t^{\prime}\right) G_{1}\left(\cdot, \eta t^{\prime}\right)(y)=W\left(\eta\left(1-t^{\prime}\right)\right)\left(D^{s} F\left(\cdot, \eta t^{\prime}\right)\right)\left(\eta^{1 / 3} y\right)$. Define

$$
\begin{aligned}
& G_{2}\left(y, t^{\prime}\right)=G_{1}\left(y, \eta t^{\prime}\right), \\
& G_{3}\left(y, t^{\prime}\right)=D^{-s} G_{2}\left(y, t^{\prime}\right) .
\end{aligned}
$$


so that

$$
\begin{aligned}
& D^{s} W\left(1-t^{\prime}\right) G_{3}\left(\cdot, t^{\prime}\right)=W\left(1-t^{\prime}\right) D^{s} G_{3}\left(\cdot, t^{\prime}\right)=W\left(1-t^{\prime}\right) G_{2}\left(\cdot, t^{\prime}\right) \\
& \quad=W\left(1-t^{\prime}\right) G_{1}\left(\cdot, \eta t^{\prime}\right)
\end{aligned}
$$

Now, we are able to apply the estimate Lemma 2.3 (4), to obtain

$$
\begin{aligned}
\eta^{1+\frac{1}{6}}\left(\int\left|\psi(1) \int_{0}^{1} W\left(\eta\left(1-t^{\prime}\right)\right)\left(D^{s} F\left(\cdot, \eta t^{\prime}\right)\right)\left(\eta^{1 / 3} y\right) d t^{\prime}\right|^{2} d y\right)^{1 / 2} \\
\quad=\eta^{1+\frac{1}{6}}\left\|\psi(1) \int_{0}^{1} W\left(1-t^{\prime}\right) G_{3}\left(\cdot, t^{\prime}\right) d t^{\prime}\right\|_{H^{s}} \leq c \eta^{1+\frac{1}{6}}\left\|G_{3}\right\|_{X^{s, b-1}} \\
=c \eta^{1+\frac{1}{6}}\left\|D^{-s} G_{2}\right\|_{X^{s, b-1}}=c \eta^{1+\frac{1}{6}}\left\|G_{2}\right\|_{X^{0, b-1}}=c \eta^{1+\frac{1}{6}}\left\|D^{s} F_{\eta}\right\|_{X^{0, b-1}} \\
=c \eta^{1+\frac{1}{6}}\left\|F_{\eta}\right\|_{X^{s, b-1}} .
\end{aligned}
$$

Therefore, we must calculate $\left\|F_{\eta}\right\|_{X^{s, b-1}}=\left\|F\left(\eta^{1 / 3} y, \eta t^{\prime}\right)\right\|_{X^{s, b-1}}$.

$$
\begin{aligned}
& \left\|F\left(\eta^{1 / 3} y, \eta t^{\prime}\right)\right\|_{X^{s, b-1}} \\
& \left.\quad=\left.\left(\int_{\mathbb{R}} \int_{\mathbb{R}}(1+|\tau-\xi|)^{2(b-1)}(1+|\xi|)^{2 s} \mid F\left(\widehat{\eta^{1 / 3} y, \eta t^{\prime}}\right)(\xi, \tau)\right)\right|^{2} d \xi d \tau\right)^{1 / 2} .
\end{aligned}
$$

Since $F\left(\widehat{\eta^{1 / 3} y}, \eta t^{\prime}\right)(\xi, \tau)=\eta^{-4 / 3} \hat{F}\left(\eta^{-1 / 3} \xi, \eta^{-1} \tau\right)$,

$$
\begin{aligned}
& \left\|F\left(\eta^{1 / 3} y, \eta t^{\prime}\right)\right\|_{X^{s, b-1}} \\
& \quad=\left(\int_{\mathbb{R}} \int_{\mathbb{R}}(1+|\tau-\xi|)^{2(b-1)}(1+|\xi|)^{2 s}\left|\eta^{-4 / 3} \hat{F}\left(\eta^{-1 / 3} \xi, \eta^{-1} \tau\right)\right|^{2} d \xi d \tau\right)^{1 / 2} .
\end{aligned}
$$

With the change of variables, $\xi^{\prime}=\eta^{-1 / 3} \xi, \tau^{\prime}=\eta^{-1} \tau$, the above expression simplifies as follows

$$
\begin{aligned}
\| F & \left(\eta^{1 / 3} y, \eta t^{\prime}\right) \|_{X^{s, b-1}} \\
& =\left(\int_{\mathbb{R}} \int_{\mathbb{R}}\left(1+\eta\left|\tau^{\prime}-\xi^{\prime}\right|\right)^{2(b-1)}\left(1+\eta^{1 / 3}\left|\xi^{\prime}\right|\right)^{2 s}\left|\hat{F}\left(\xi^{\prime}, \tau^{\prime}\right)\right|^{2} \eta^{-8 / 3} \eta^{4 / 3} d \xi^{\prime} d \tau^{\prime}\right)^{1 / 2} \\
& =\eta^{-2 / 3}\left(\int_{\mathbb{R}} \int_{\mathbb{R}}\left(1+\eta\left|\tau^{\prime}-\xi^{\prime}\right|\right)^{2(b-1)}\left(1+\eta^{1 / 3}\left|\xi^{\prime}\right|\right)^{2 s}\left|\hat{F}\left(\xi^{\prime}, \tau^{\prime}\right)\right|^{2} d \xi^{\prime} d \tau^{\prime}\right)^{1 / 2} .
\end{aligned}
$$

Now, we must consider the following different cases,
(1) $\left\{\begin{array}{l}\eta^{1 / 3}\left|\xi^{\prime}\right| \leq 1, \\ \eta\left|\tau^{\prime}-\xi^{\prime}\right| \leq 1 .\end{array}\right.$ In this case, $\left\|F_{\eta}\right\|_{X^{s, b-1}} \leq \eta^{-2 / 3}\|F\|_{X^{s, b-1}}$.
(2) $\left\{\begin{array}{l}\eta^{1 / 3}\left|\xi^{\prime}\right| \leq 1, \\ \eta\left|\tau^{\prime}-\xi^{\prime}\right| \geq 1 .\end{array}\right.$ In this case, $\left\|F_{\eta}\right\|_{X^{s, b-1}} \leq \eta^{b-1-2 / 3}\|F\|_{X^{s, b-1}}$.
(3) $\left\{\begin{array}{l}\eta^{1 / 3}\left|\xi^{\prime}\right| \geq 1, \\ \eta\left|\tau^{\prime}-\xi^{\prime}\right| \leq 1 .\end{array}\right.$ In this case, $\left\|F_{\eta}\right\|_{X^{s, b-1}} \leq \eta^{s / 3-2 / 3}\|F\|_{X^{s, b-1}}$.

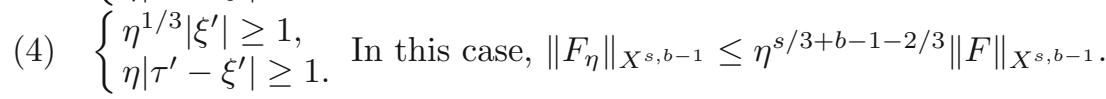


Recall that $1 / 2<b<1$. We use the more restrictive upper bound which corresponds to the second case to conclude that the estimate (2.48) remains as follows:

$$
\begin{aligned}
& c \eta^{1+1 / 6}\left\|F_{\eta}\right\|_{X^{s, b-1}} \leq c \eta^{1+1 / 6+b-1-2 / 3}\|F\|_{X^{s, b-1}} \\
& \quad=c \eta^{b-1 / 2}\|F\|_{X^{s, b-1}} .
\end{aligned}
$$

Then, (2.41) goes to 0 when $\eta \rightarrow 0$ and, using the continuity of the free operator $W(t)$,

$$
\lim _{t \rightarrow 0}\left\|w(t)-\psi(t) W(t) w_{0}\right\|_{H^{s}}=0
$$

4. We now prove the persistence property (2.13), i.e., $w \in C\left([-1,1]: H^{s}(\mathbb{R})\right)$. First, we need to prove the continuity of the norm of the $X^{s, b}$ space but this follows a similar argument as in the proof of the continuity of the initial data in the $H^{s}$ norm 2.40, therefore it will be omitted. From this, the persistence property in $H^{s}$ is a direct consequence of the embedding $X^{s, b} \subset C_{t} H^{s}$ for $b>1 / 2$ (see [p.156, corollary 7.3][12]).

Proof of Theorem 2.2. From the unicity and local existence, at time $t=T>0$ of the IVP (2.2) for $w$, we get unicity and local existence, at time $T=\lambda^{-3}$, of the IVP (1.1) for $v$, whenever we determine for which values of $\lambda$, depending on $\left\|v_{0}\right\|_{H^{s}}$, conditions (2.30a) and (2.30b) are verified. In this way, we must calculate the norm $r_{w}=\left\|w_{0}\right\|_{H^{s}}$, taking now into account that $w_{0}(x)=$ $\lambda^{-\alpha} v_{0}\left(\lambda^{-1} x\right)$, using (2.1). By definition

$$
r_{w}=\left\|w_{0}\right\|_{H^{s}}=\left(\left.\int_{\mathbb{R}}(1+|\xi|)^{2 s} \widehat{\mid w_{0}(x)}(\xi)\right|^{2} d \xi\right)^{1 / 2}
$$

where,

$$
\begin{aligned}
\widehat{w_{0}(x)}(\xi) & =\int_{\mathbb{R}} e^{-i x \xi} w_{0}(x) d x=\int_{\mathbb{R}} e^{-i x \xi} \lambda^{-\alpha} v_{0}\left(\lambda^{-1} x\right) d x \\
& =\int_{\mathbb{R}} e^{-i\left(\lambda^{-1} x\right) \lambda \xi} \lambda^{-\alpha} v_{0}\left(\lambda^{-1} x\right) \lambda d\left(\lambda^{-1} x\right) \\
& =\lambda^{1-\alpha} \int_{\mathbb{R}} e^{-i\left(\lambda^{-1} x\right) \lambda \xi} v_{0}\left(\lambda^{-1} x\right) d\left(\lambda^{-1} x\right) \\
& =\lambda^{1-\alpha} \hat{v}_{0}(\lambda \xi) .
\end{aligned}
$$

Then, (2.54) is rewritten as follows

$$
\begin{aligned}
r_{w} & =\left(\int_{\mathbb{R}}(1+|\xi|)^{2 s}\left|\widehat{w_{0}(x)}(\xi)\right|^{2} d \xi\right)^{1 / 2}=\left(\int_{\mathbb{R}}(1+|\xi|)^{2 s}\left|\lambda^{1-\alpha} \hat{v}_{0}(\lambda \xi)\right|^{2} d \xi\right)^{1 / 2} \\
& =\left(\lambda^{1-\alpha} \int_{\mathbb{R}}\left(1+\lambda^{-1}|\lambda \xi|\right)^{2 s}\left|\hat{v}_{0}(\lambda \xi)\right|^{2} \lambda^{-1} d(\lambda \xi)\right)^{1 / 2} \\
& =\lambda^{1-1 / 2-\alpha}\left(\int_{\mathbb{R}}\left(1+\lambda^{-1}|\lambda \xi|\right)^{2 s}\left|\hat{v}_{0}(\lambda \xi)\right|^{2} d(\lambda \xi)\right)^{1 / 2} \\
& =\lambda^{1 / 2-\alpha}\left(\int_{\mathbb{R}}\left(1+\lambda^{-1}|\lambda \xi|\right)^{2 s}\left|\hat{v}_{0}(\lambda \xi)\right|^{2} d(\lambda \xi)\right)^{1 / 2} .
\end{aligned}
$$


Now denoting $r_{v}=\left\|v_{0}\right\|_{H^{s}}$, the norm $r_{w}=\left\|w_{0}\right\|_{H^{s}}$ can be estimated in terms of $r_{v}$, splitting first the last integral in (2.55) and estimating next:
(a) $\lambda^{1 / 2-\alpha}\left(\int_{\lambda^{-1}|\eta| \leq 1}\left|v_{0}(\eta)\right|^{2} d \eta\right)^{1 / 2} \leq \lambda^{1 / 2-\alpha}\left\|v_{0}\right\|_{L^{2}(\mathbb{R})} \leq \lambda^{1 / 2-\alpha} r_{v}$
(b) $\lambda^{1 / 2-\alpha-s}\left(\int_{\lambda^{-1}|\eta| \geq 1}|\eta|^{2 s}\left|v_{0}(\eta)\right|^{2} d \eta\right)^{1 / 2} \leq \lambda^{1 / 2-\alpha-s}\left\|v_{0}\right\|_{\dot{H}^{s}(\mathbb{R})}$
$\leq \lambda^{1 / 2-\alpha-s} r_{v}$

Now, taking into account the latter bounds for the norm $r_{w}$ when $\lambda^{-1}|\eta| \leq 1$ and $\lambda^{-1}|\eta| \geq 1$ respectively and recalling that $s>1 / 4$, we determine for which values of $\lambda$ the conditions (2.30) are satisfied.

(i) Condition (2.30a): $r_{w} \lambda^{\alpha-2} c_{0} \leq 1 / 4$.

(i.a)

$$
\lambda^{1 / 2-\alpha} r_{v} \lambda^{\alpha-2} c_{0} \leq 1 / 4 \rightarrow \lambda^{-3 / 2} \leq \frac{1}{4 c_{0} r_{v}}
$$

$$
\lambda^{1 / 2-\alpha-s} r_{v} \lambda^{\alpha-2} c_{0} \leq 1 / 4 \rightarrow \lambda^{-3 / 2-s} \leq \frac{1}{4 c_{0} r_{v}} .
$$

(ii) Condition (2.30b): $r_{w}^{2} \lambda^{2(\alpha-1)} c_{0}^{2} \leq 1 / 4$.

(ii.a)

$$
\lambda^{1-2 \alpha} r_{v}^{2} \lambda^{2(\alpha-1)} c_{0}^{2} \leq 1 / 4 \rightarrow \lambda^{-1} \leq \frac{1}{4 c_{0}^{2}\left(r_{v}\right)^{2}}
$$

$$
\lambda^{1-2 \alpha-2 s} r_{v}^{2} \lambda^{2(\alpha-1)} c_{0}^{2} \leq 1 / 4 \rightarrow \lambda^{-1-2 s} \leq \frac{1}{4 c_{0}^{2}\left(r_{v}\right)^{2}} .
$$

In this way, we distinguish two different cases:

1.

$$
\frac{1}{4 c_{0} r_{v}} \leq 1
$$

From (i), if (i.a) is satisfied, (i.b) will also be satisfied. Then,

$$
\lambda^{-3 / 2} \leq \frac{1}{4 c_{0} r_{v}} .
$$

From (ii), if (ii.a) is satisfied, (ii.b) will also be satisfied. So that

$$
\lambda^{-1} \leq \frac{1}{4 c_{0}^{2}\left(r_{v}\right)^{2}} \Rightarrow \lambda^{-3 / 2} \leq \frac{1}{2 c_{0}^{2} r_{v}^{2}\left(4 c_{0} r_{v}\right)}
$$

Comparing these two estimates on $\lambda$, the most restrictive is

$$
\lambda^{-1} \leq \frac{1}{4 c_{0}^{2}\left(r_{v}\right)^{2}}
$$


2.

$$
\frac{1}{4 c_{0}^{2}\left(r_{v}\right)^{2}} \geq 1
$$

From (i), if (i.b) is satisfied, (i.a) will also be satisfied. Therefore,

$$
\lambda^{-3 / 2-s} \leq \frac{1}{4 c_{0} r_{v}} .
$$

From (ii), if (ii.b) is satisfied, (ii.a) will also be satisfied. Then,

$$
\lambda^{-1-2 s} \leq \frac{1}{4 c_{0}^{2}\left(r_{v}\right)^{2}} .
$$

From these two estimates on $\lambda$, the most restrictive is

$$
\lambda^{-3 / 2-s} \leq \frac{1}{4 c_{0} r_{v}} .
$$

Hence, whenever $\lambda$ satisfies (2.57) or (2.58), the application $\Phi_{T=\lambda^{-3}}$ is contractive in $\mathcal{B}_{v}$, the ball associated to the ball $\mathcal{B}$ given in (2.28) through the scaling relation. So, there exists a unique $v \in \mathcal{B}_{v}$ solution of the IVP (1.1). Note that the local existence time is explicit from the relation $\lambda T=1$ and from the values of $\lambda(2.57)$ and (2.58). That is,

$$
\begin{aligned}
& T=\lambda^{-3} \leq\left(\frac{1}{4 c_{0}^{2}\left\|v_{0}\right\|_{H^{s}}^{2}}\right)^{3}, \quad\left\|v_{0}\right\|_{H^{s}} \geq 1, \\
& T=\lambda^{-3} \leq\left(\frac{1}{4 c_{0}\left\|v_{0}\right\|_{H^{s}}}\right)^{\frac{6}{3+2 s}}, \quad\left\|v_{0}\right\|_{H^{s}} \leq 1 .
\end{aligned}
$$

With the same argument used in (2.37), it verifies that

$$
\|v-\tilde{v}\|_{X^{s, b}} \leq c\left\|v_{0}-\tilde{v}_{0}\right\|_{H^{s}} .
$$

The property of persistence (2.13) for $v$, i.e.,

$$
v \in C\left([-T, T]: H^{s}(\mathbb{R})\right),
$$

follows directly from the property of persistence for the auxiliar function $w$. This complete the proof.

\subsection{Global theory}

The global well-posedness for the IVP of the GE (1.1) with initial data in $H^{1}(\mathbb{R})$ is stated in the following theorem:

Theorem 2.7. Let be $u_{0} \in H^{1}(\mathbb{R})$ and $u$ the corresponding local solution for the IVP of the GE (1.1) given by the Theorem 2.2. Then we extend the solution for all $t>0$, that is

$$
u \in C\left(\mathbb{R}: H^{1}(\mathbb{R})\right) .
$$

Proof. It follows using the local existence result and standard techniques of Gagliardo-Nirenberg inequalities and conservation of the energy. 


\section{Stability of solitons}

We consider here the question of the stability of solitons of the Gardner equation (1.1) under small perturbations in $H^{1}(\mathbb{R})$. Since the $\mathrm{mKdV}$ and Gardner equations are closely related, this question is equivalent to study the stability under small perturbations in $H^{1}(\mathbb{R})$ of solitons with non bounded mean of the focusing and defocusing $\mathrm{mKdV}$ (positive/negative nonlinearity, respectively),

$$
\frac{\partial}{\partial t} k(x, t)+\frac{\partial}{\partial x^{3}} k(x, t) \pm 2 \frac{\partial}{\partial x}\left(k^{3}(x, t)\right)=0 .
$$

In this section, we compute necessary conditions, given by Zhidkov (see [15]) in a general framework, to obtain the stability of solitons. In such work, Zhidkov states a general theorem about the stability of solitons of the gKdV equation in $H^{2}(\mathbb{R})$, vanishing in the boundary. In our case, the stability result is centered on the stability of solitons of the Gardner equation.

\subsection{Existence of travelling wave solutions}

We look for solutions of the focusing $\mathrm{mKdV}$

$$
\frac{\partial}{\partial t} k(x, t)+\frac{\partial}{\partial x^{3}} k(x, t)+2 \frac{\partial}{\partial x}\left(k^{3}(x, t)\right)=0
$$

of the following type

$$
k(x, t)=\sigma+\phi\left(x-c_{\sigma} t\right), \quad c_{\sigma}>0, \phi( \pm \infty)=0 .
$$

Introducing this ansatz in (3.2), we obtain $-c_{\sigma} \phi^{\prime}+\phi^{\prime \prime}+2\left((\sigma+\phi)^{3}\right)^{\prime}=0$. Integrating and taking into account that $\phi( \pm \infty)=0$, we arrive to

$$
\left\{\begin{array}{l}
\phi^{\prime \prime}+2 \phi^{3}+6 \sigma \phi^{2}+\left(6 \sigma^{2}-c_{\sigma}\right) \phi=0 \\
\phi( \pm \infty)=0
\end{array}\right.
$$

Now multiplying (3.4) by the integrating factor $\phi^{\prime}$, we arrive to

$$
\left(\phi^{\prime}\right)^{2}+\phi^{4}+4 \sigma \phi^{3}+\left(6 \sigma^{2}-c_{\sigma}\right) \phi^{2}=0 .
$$

This ODE can be solved explicitly and we obtain (see Fig. 1)

$$
\begin{aligned}
& \phi_{\sigma, c_{0}}\left(x-c_{\sigma}\left(\sigma, c_{0}\right) t\right)=\frac{c_{0}}{2 \sigma+\sqrt{4 \sigma^{2}+c_{0}} \cosh \left(\sqrt{c_{0}}\left(x-\left(6 \sigma^{2}+c_{0}\right) t\right)\right.} . \\
& c_{\sigma}\left(\sigma, c_{0}\right)=6 \sigma^{2}+c_{0} .
\end{aligned}
$$

We can also characterize the soliton of the Gardner equation as the minimum of the following Lyapunov functional: if we denote

$$
\begin{aligned}
& \mathcal{E}(f)=\int_{\mathbb{R}}\left\{f_{x}^{2}-f^{4}-4 \sigma f^{3}\right\} d x, \\
& \mathcal{F}(f)=\frac{1}{2} \int_{\mathbb{R}} f^{2} d x,
\end{aligned}
$$

as the energy and the $L^{2}$ norm of the soliton of the Gardner equation (2.2), perturbing the Lyapunov functional (3.8)

$$
E(\xi)=\mathcal{E}(\xi)+2\left(c_{\sigma}-6 \sigma^{2}\right) \mathcal{F}(\xi)=\int_{\mathbb{R}}\left\{\xi_{x}^{2}-\xi^{4}-4 \sigma \xi^{3}+\left(c_{\sigma}-6 \sigma^{2}\right) \xi^{2}\right\} d x,
$$



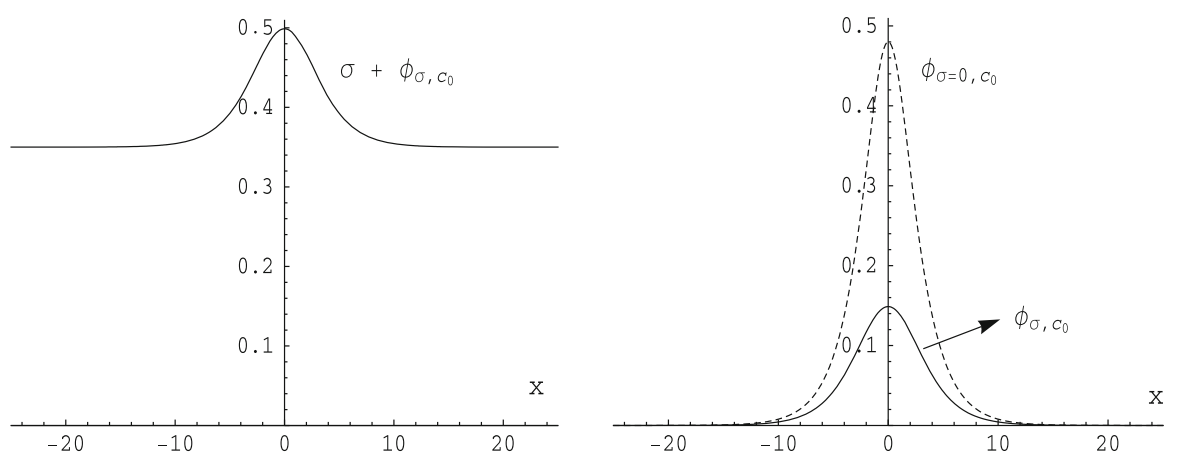

Figure 1. Left Profile of $\sigma+\phi_{\sigma, c_{0}}$ with $\sigma=0.35, c_{0}=0.23$. Right Comparison between humps of the soliton of $\mathrm{mKdV}$ (dashed line, $\phi_{\sigma=0, c_{0}}$ ) and the soliton of GE (solid line, $\phi_{\sigma, c_{0}}$ ) with $\sigma=0.35$, and both with $c_{0}=0.23$.

around its critical point, we obtain the linearized operator $L$ and the ODE satisfied by the soliton of the Gardner equation,

$$
\begin{aligned}
& L=-\partial_{x x}+c_{\sigma}-6\left(\sigma+\phi_{\sigma, c_{0}}\right)^{2} . \\
& -\phi_{\sigma, c_{0}}^{\prime \prime}-2 \phi_{\sigma, c_{0}}^{3}-6 \sigma \phi_{\sigma, c_{0}}^{2}+\left(c_{\sigma}-6 \sigma^{2}\right) \phi_{\sigma, c_{0}}=0 .
\end{aligned}
$$

The main results of this section are the following:

Theorem 3.1. (focusing case) Let $\sigma \in \mathbb{R}, c_{0} \in(0, \infty)$ and $u_{\sigma, c_{0}}(x, t)=\sigma+$ $\phi_{\sigma, c_{0}}\left(x-c_{\sigma}\left(\sigma, c_{0}\right) t\right) \in \dot{H}^{1}(\mathbb{R}), c_{\sigma}\left(\sigma, c_{0}\right)=c_{0}+6 \sigma^{2}$, a solution of the focusing $m K d V(3.2)$, where $\phi_{\sigma, c_{0}} \in H^{1}(\mathbb{R})$ satisfies (3.4). Then $\forall \epsilon>0, \exists \delta \equiv$ $\delta\left(\epsilon, \sigma, c_{0}\right)>0$ and a function $C^{2}(\mathbb{R}), r: \mathbb{R} \rightarrow \mathbb{R}$, such that if $\| u_{0}-(\sigma+$ $\left.\phi_{\sigma, c_{0}}\right) \|_{H^{1}(\mathbb{R})}<\delta$, then

$$
\sup _{t>0}\left\|u(\cdot, t)-u_{\sigma, c_{0}}(\cdot+r(t))\right\|_{H^{1}(\mathbb{R})}<\epsilon,
$$

where $u(x, t)$ is the unique solution of the focusing $m K d V$ equation with initial data $u_{0}=u(x, 0) \in \dot{H}^{1}(\mathbb{R})$ and where $\sup _{t}\left|r^{\prime}(t)+\left(c_{0}+6 \sigma^{2}\right)\right| \leq K \epsilon, K>0$.

Theorem 3.2. (defocusing case). Let $\sigma \in \mathbb{R}, c_{0} \in\left(0,4 \sigma^{2}\right)$ and $u_{\sigma, c_{0}}(x, t)=$ $\sigma-\varphi_{\sigma, c_{0}}\left(x+c_{\sigma}\left(\sigma, c_{0}\right) t\right) \in \dot{H}^{1}(\mathbb{R}), c_{\sigma}\left(\sigma, c_{0}\right)=6 \sigma^{2}-c_{0}$, a solution of the defocusing $m K d V$, where $\varphi_{\sigma, c_{0}} \in H^{1}(\mathbb{R})$ is solution of the defocusing Gardner equation. Then $\forall \epsilon>0, \exists \delta \equiv \delta\left(\epsilon, \sigma, c_{0}\right)>0$ and a function $C^{2}(\mathbb{R}), r: \mathbb{R} \rightarrow \mathbb{R}$, such that if $\left\|u_{0}-\left(\sigma-\varphi_{\sigma, c_{0}}\right)\right\|_{H^{1}(\mathbb{R})}<\delta$, then

$$
\sup _{t>0}\left\|u(\cdot, t)-u_{\sigma, c_{0}}(\cdot+r(t))\right\|_{H^{1}(\mathbb{R})}<\epsilon,
$$

where $u(x, t)$ is the unique solution of the focusing $m K d V$ equation with initial data $u_{0}=u(x, 0) \in \dot{H}^{1}(\mathbb{R})$ and where $\sup _{t}\left|r^{\prime}(t)+\left(c_{0}-6 \sigma^{2}\right)\right| \leq K \epsilon, K>0$.

Before explaining the main ideas behind the proof of this result, some remarks are in order. 
Remarks. (i) We will fix the parameter $\sigma$ and we will choose the parameter $c_{0}$ in the interval $c_{0} \in(0, \infty)$ since we are only interested in real regular solutions. In this way, the application

$$
c_{0} \in(0, \infty) \stackrel{\Phi}{\longrightarrow} \phi_{\sigma, c_{0}} \in H^{1}(\mathbb{R})
$$

is $C^{1}\left(\mathbb{R}^{+}: H^{1}(\mathbb{R})\right)$.

(ii) The soliton solution of the defocusing $\mathrm{mKdV}$ equation is easily obtained from the explicit expression (3.6) in the focusing case, with the change $\sigma$ to $i \sigma$.

Proof of Theorem 3.1. We use the standard techniques given by Weinstein [14], Zhidkov [15] and Angulo [3,4] and then we do not give the details here. We only comment two main results in the proof:

1. Convexity of the function $d\left(c_{0}\right): d^{\prime \prime}\left(c_{0}\right)>0, \forall c_{0} \in(0, \infty)$, where

$$
d\left(c_{0}\right)=\int_{\mathbb{R}}\left\{\partial_{x}^{2} \phi_{\sigma, c_{0}}-\phi_{\sigma, c_{0}}^{4}-4 \sigma \phi_{\sigma, c_{0}}^{3}+\left(c_{\sigma}-6 \sigma^{2}\right) \phi_{\sigma, c_{0}}^{2}\right\} d x .
$$

Since the Gardner equation is not invariant under scaling transformations, we can not proceed as usual to prove the convexity of the function (3.12). But we know explicitly the soliton solution $\phi_{\sigma, c_{0}}$ of the Gardner equation [see (3.6)] and then by its relative simplicity, we can integrate directly (maybe with the help of a handbook of integrals, e.g., [8]) obtaining

$$
d^{\prime \prime}\left(c_{0}\right)=2 \int_{\mathbb{R}} \phi_{\sigma, c_{0}} \partial_{c_{0}} \phi_{\sigma, c_{0}} d s=\frac{\sqrt{c_{0}}}{4 \sigma^{2}+c_{0}}>0, \quad \forall c_{0} \in(0, \infty) .
$$

2. The phase $r(t)$ and its velocity $r^{\prime}(t)$. Define the function $F: \mathbb{R}^{2} \rightarrow \mathbb{R}$ given by,

$$
\begin{aligned}
F(r, t) & =\frac{1}{2} \int_{\mathbb{R}}\left\{u(x, t)-\left(\sigma+\phi_{\sigma, c_{0}}(x+r)\right)\right\}^{2} d x \\
& =\frac{1}{2} \int_{\mathbb{R}}\left\{\psi(x, t)-\phi_{\sigma, c_{0}}(x+r)\right\}^{2} d x .
\end{aligned}
$$

Since $\phi_{\sigma, c_{0}} \in H^{\infty}(\mathbb{R}), F$ is a $C^{\infty}$ function in the $r$ variable and $C^{1}$ in the $t$ variable. With these properties for $F$ we define the following associated function,

$$
\begin{aligned}
G(r, t) & =\frac{\partial F}{\partial r}=-\int_{\mathbb{R}}\left\{u(x, t)-\left(\sigma+\phi_{\sigma, c_{0}}(x+r)\right)\right\} \partial_{x} \phi_{\sigma, c_{0}}(x+r) d x \\
& =-\int_{\mathbb{R}} u(x, t) \partial_{x} \phi_{\sigma, c_{0}}(x+r) d x .
\end{aligned}
$$

It is easy to see that $G(0,0)=0$ and $\left.\frac{\partial G}{\partial r}\right|_{(0,0)} \geq 0$. Then, applying the implicit function theorem to the function $G$, there exist $T>0$ and a $C^{2}((-T, T): \mathbb{R})$, function $r:(-T, T) \rightarrow \mathbb{R}, r(0)=0$, such that (from $G$ and its time derivatives, we check that the function $r(t)$ is $C^{2}$ )

$$
G(r(t), t)=0, \quad \forall t \in(-T, T) .
$$


We choose $T$ in a maximal way. Then derivating implicitly (3.15), we get

$$
\begin{aligned}
\frac{d}{d t} G(r(t), t)=0 & =\frac{\partial G}{\partial r} r^{\prime}(t)+\frac{\partial G}{\partial t} \Rightarrow \\
r^{\prime}(t) & =-\frac{\frac{\partial G}{\partial t}}{\frac{\partial G}{\partial r}} .
\end{aligned}
$$

Then, once we calculate $\frac{\partial G}{\partial t}, \frac{\partial G}{\partial r}$, and substituting in (3.16), we obtain the ODE satisfied by the phase $r(t)$

$$
\left\{\begin{array}{l}
r^{\prime}(t)=-\left(c_{0}+6 \sigma^{2}\right)-\frac{\int_{\mathbb{R}} h\left\{-12 v\left(v^{\prime}\right)^{2}+6 h v v^{\prime \prime}+2 h^{2} v^{\prime \prime}\right\} d x}{\int_{\mathbb{R}}\left\{-\left(v^{\prime}\right)^{2}+h v^{\prime \prime}\right\}}, \\
r(0)=0
\end{array}\right.
$$

where, $h(x, t)=u(x, t)-\left(\sigma+\phi_{\sigma, c_{0}}(x+r(t))\right)$ and $v(x, t)=\sigma+\phi_{\sigma, c_{0}}$ $(x+r(t))$.

Proof of Theorem 3.2. It follows the same steps than in the focusing case (up to the obvious change in the linearized operator and the speed of the soliton), with the important difference of the existence interval for the parameter $c_{0} \in\left(0,4 \sigma^{2}\right)$.

\section{Acknowledgments}

The author wishes to express his sincere thanks to Professor Luis Vega for his continuous encouragement and useful discussions during the elaboration of this work.

\section{References}

[1] Alejo, M.A.: Geometric breathers of the mKdV equation (submitted)

[2] Alejo, M.A., Muñoz, C., Vega, L.: The Gardner equation and the $L^{2}$-stability of the $N$-soliton solution of the Korteweg-de Vries equation. Trans. Am. Math. Soc. (to appear)

[3] Angulo, J.: Stability of Cnoidal waves. Adv. Differ. Equ. 11(12), 1312-1374 (2006)

[4] Angulo, J.: Nonlinear stability of periodic traveling wave solutions to the Schrödinger and the modified Korteweg de Vries equations. J. Differ. Equ. 235, 1-30 (2007)

[5] Bourgain, J.: Fourier transform restriction phenomena for certain lattice subsets and applications to nonlinear evolution equations. Geom. Funct. Anal. 3, 107-156, 209-262 (1993)

[6] Christ, M., Colliander, J., Tao, T.: Asymptotics, frequency modulation, and low regularity ill-posedness for canonical defocusing equations. Am. J. Math. 125, 1235-1293 (2003) 
[7] Colliander, J., Keel, M., Staffilani, G., Takaoka, H., Tao, T.: Sharp global wellposedness results for periodic and non-periodic $\mathrm{KdV}$ and modified $\mathrm{KdV}$ on $\mathbb{R}$ and $\mathbb{T}$. JAMS 16, 705-749 (2003)

[8] Gradshtein, I.S., Ryzhik, I.M.: Tables of integrals, series and products, Academic Press, New York (1969)

[9] Kenig, C., Ponce, G., Vega, L.: Well-posedness and scattering results for the generalized Korteweg-de Vries equation via the contraction principle. Commun. Pure Appl. Math. 46(4), 527-620 (1993)

[10] Kenig, C., Ponce, G., Vega, L.: The Cauchy problem for the KdV equation in Sobolev spaces of negative indices. Duke Math. J. 71(1), 1-20 (1993)

[11] Kenig, C., Ponce, G., Vega, L.: A bilinear estimate with applications to the KdV equation. J. Am. Math. Soc. 8(2), 573-603 (1996)

[12] Linares, F., Ponce, G.: Introduction to nonlinear dispersive equations. Springer, Berlin (2000)

[13] Tao, T.: Multilinear weighted convolution of $L^{2}$ functions, and applications to non-linear dispersive equations. Am. J. Math. 123, 839-908 (2001)

[14] Weinstein, M.I.: Lyapunov stability of ground states of nonlinear dispersive equations. Commun. Pure Appl. Math. 39, 51-68 (1986)

[15] Zhidkov, P.E.: Korteweg-de Vries and Nonlinear Schrödinger Equations: Qualitative Theory. In: Lecture Notes in Mathematics, vol. 1756. Springer, Berlin (2001)

Miguel A. Alejo $(\varangle)$

Department of Mathematics

University of the Basque Country

CP 48940, Leioa

Spain

e-mail: miguelangel.alejo@ehu.es

Received: 24 January 2011.

Accepted: 20 September 2011. 